ISSN 1112-9867

\title{
ABOUT ONE NON LINEAR GENERALIZATION OF THE COMPRESSION REFLECTION PRINCIPLE
}

$$
\text { . . Vantsyan }{ }^{1, *}, \text { H. . . Gevorgyan }{ }^{2} \text { and M. Moravej }{ }^{3}
$$

${ }^{1}$ Chief researcher of Institute of Mechanics of National Academy of Sciences of the Republic of Armenia

${ }^{2}$ Researcher of Institute of mechanics of National Academy of Sciences of the Republic of Armenia

${ }^{3}$ Department of Engineering, Payame Noor Universtiy, PO BOX 19395-3697 Tehran, Iran

Published online: 05 June 2016

\begin{abstract}
Generalize method of iteration is proposed, presented as a combination of the classical iteration and proportional division methods, on which the conditions of Boltsano-Cochy theorems are satisfied. n evidence of the proposed algorithm's convergence is brought. Originally, the compression reflection operator as a function from one variable is considered. A theorem about the convergence of this iteration and its evidence are brought. Both cases of stage and spiral iterations are considered. A geometrical interpretation of a convergence of a generalize method of iteration is brought, the case of stage and spiral iterations are considered. The formula for the non linear generalize compression reflection operator as a function from one variable is obtained. A formulation of the non linear generalizes reflections as a function from some variables is also exposed. On the exercises of some examples of the transcendent equations systems the advantage of a generalize method of iteration on respect to the known methods a view point as of the width of region as of the speed of convergence was showed.
\end{abstract}

Author Correspondence, e-mail: vantsyan@mechins.sci.am

doi: http://dx.doi.org/10.4314/jfas.8vi2s.51 
Keywords: generalize method of iteration; non linear generalize reflection; compressing original; convergence; immovable point; transcendent equations systems.

\section{INTRODUCTION}

In the publications $[1-3]$ the detailing foundation of a theoretical existence and applicable efficiency of the generalize method of iteration (GMI) used for the solution of a non linear algebraic and transcendent equations was given. Moreover, in the work [ 3 ] was showed, that the GMI put forward as a limit stage of an analytical development of the classical and the modernize [ 3 ] iteration methods and intends for an effective numerical solution of the non linear algebraic, transcendent and differential equations as well as the equations systems called classes of non linear equations.

In the present article is supposed enlarge partly the traditional presentations about compression reflection [ 4 ] as a fundamental principle of a solution of the systems of algebraic, transcendent and differential equations by the method of successive approximations.

The new non linear generalization of the compression reflections principle serves the theoretical foundation for numerical solution of the non linear equations systems of by the GMI.

\section{ORIGINAL IDEA OF GENERALIZATION OF THE METHOD OF ITERATIA}

As an illustration of the initial idea of generalization of the iteration method, first of all, is brought the algorithm of a numerical solution for non linear algebraic equations by modernize method of iteration (MMI) (Fig. 1, a) [1, 2]:

1. Input of an initial approximation $\mathrm{x}_{0} \in[\mathrm{a}, \mathrm{b}]$;

2. Calculation $\mathrm{x}_{1}=\phi\left(\mathrm{x}_{0}\right)$;

3. Find the $\mathrm{x}_{2}=\frac{\mathrm{x}_{0}+\mathrm{x}_{1}}{2}$;

4. Calculation $\mathrm{x}_{3}=\phi\left(\mathrm{x}_{2}\right)$;

5. Find the $\mathrm{x}_{4}=\frac{\mathrm{x}_{2}+\mathrm{x}_{3}}{2}$ и т.д.

The principal scheme of the given algorithm is brought on the Fig. 1, (a). 
For evidence of the process of convergence the known estimation is carried out [5]:

1. $\left|\mathrm{x}_{1}-\xi\right|=\left|\phi\left(\mathrm{x}_{0}\right)-\phi(\xi)\right| \leq \mathrm{q}\left|\mathrm{x}_{0}-\xi\right|, \quad|\mathrm{q}|<1$

2. $\left|\mathrm{x}_{2}-\xi\right|=\left|\frac{\mathrm{x}_{0}+\mathrm{x}_{1}}{2}-\xi\right|=\frac{1}{2}\left|\left(\mathrm{x}_{0}-\xi\right)+\left(\mathrm{x}_{1}-\xi\right)\right| \leq$

$$
\begin{aligned}
& \leq \frac{1}{2}\left|\mathrm{x}_{0}-\xi\right|+\frac{1}{2}\left|\mathrm{x}_{1}-\xi\right| \leq \frac{1}{2}\left|\mathrm{x}_{0}-\xi\right|+\frac{1}{2} \mathrm{q}\left|\mathrm{x}_{0}-\xi\right|= \\
& =\frac{1}{2}(\mathrm{q}+1)\left|\mathrm{x}_{0}-\xi\right|,
\end{aligned}
$$

after which it's not difficult to find a new estimation for the $\mathrm{n}-$ th iteration, i.e.

$$
\begin{aligned}
& \left|\mathrm{x}_{\mathrm{n}}-\xi\right| \leq \frac{1}{2^{\mathrm{n} / 2}}(\mathrm{q}+1)^{\mathrm{n} / 2}\left|\mathrm{x}_{0}-\xi\right|, \quad \text { если } \mathrm{n}=2 \mathrm{k}, \quad \mathrm{k}=0,1,2, \ldots ; \\
& \left|\mathrm{x}_{\mathrm{n}}-\xi\right| \leq \frac{1}{2^{(\mathrm{n}-1) / 2}}(\mathrm{q}+1)^{(\mathrm{n}-1) / 2}\left|\mathrm{x}_{0}-\xi\right|, \quad \text { если } \mathrm{n}=2 \mathrm{k}+1, \quad \mathrm{k}=0,1,2, \ldots
\end{aligned}
$$

In the case of a large integer number $n$ the last two estimations one can change by two expressions

$$
\left|x_{n}-\xi\right| \leq \lim _{n \rightarrow \infty}\left[\frac{1}{2^{n / 2-1 / 2}}(q+1)^{n / 2-1 / 2}\right]\left|x_{0}-\xi\right|=\lim _{n \rightarrow \infty}\left(\frac{q+1}{2}\right)^{n / 2}\left|x_{0}-\xi\right| .
$$

Because of $|\mathrm{q}|<1$, that from it fallow that, if $\left|\frac{\mathrm{q}+1}{2}\right|<1$, i.e. at $-3<\mathrm{q}<1$, the modernize method of iteration converge.

\section{NON LINEAR GENERALIZE REFLECTION AS A FUNCTION FROM ONE VARIABLE}

As an aim of to obvious illustration of a new proposed generalization of the compression reflections operator let us consider it's, before, in the form of simple function from one variable. 
As it's known [ 4 ], an arbitrary function $\varphi=\varphi(x)$, satisfying to condition $\varphi(x)=x$, used to call the compression reflection, if $\left|\varphi\left(\mathrm{x}_{1}\right)-\varphi\left(\mathrm{x}_{2}\right)\right| \leq \mathrm{q}\left|\mathrm{x}_{1}-\mathrm{x}_{2}\right| \quad$ при $0<\mathrm{q}<1$.

Let us consider the following expression as a generalize compression reflection [3]:

$\Phi(\mathrm{x})=\frac{\psi(\mathrm{x})+(\lambda-1) \mathrm{x}}{\lambda}$,

After witch it's proved, that it's practically for an arbitrary function $\psi=\psi(x)$ in the complete metric space $\boldsymbol{R}$ can be represented as a classical compression reflection, i.e. $\left|\Phi^{\prime}(\mathrm{x})\right| \leq \mathrm{q}<1$.

Theorem. Let us the function $\varphi(x)$ is continuous and differentiable on the piece $[a, b]$, moreover all its values $\varphi(x) \in[a, b]$. Then it is existed the correct fraction $q$ such that

$$
\frac{\left|\lambda-1+\varphi^{\prime}(x)\right|}{\lambda} \leq q<1
$$

at $a<x<b$, and $\lambda \in R$, the generalize iteration process

$$
x_{n}=\frac{\varphi\left(x_{n-1}\right)+(\lambda-1) x_{n-1}}{\lambda}, \quad n=1,2, \ldots
$$

to be converge, independent from original values $x_{0} \in[a, b]$ to limit value

$$
\xi=\lim _{n \rightarrow \infty} x_{n}
$$

which appears the united root of the equation

$$
f(x)=0 \text {, or } \varphi(x)=x,
$$

on the piece $[a, b]$, where carried out the conditions of the theorem Bolzano-Cauchy; $f(a) \cdot f(b)<0$ about existence of the separate isolating root (3.4) of equation (3.5).

Evidence. Let us consider two consistently approximations. 


$$
x_{n}=\frac{\varphi\left(x_{n-1}\right)+(\lambda-1) x_{n-1}}{\lambda} \text { and } \quad x_{n+1}=\frac{\varphi\left(x_{n}\right)+(\lambda-1) x_{n}}{\lambda},
$$

from which

$$
\varphi\left(x_{n}\right)-\varphi\left(x_{n-1}\right)+(\lambda-1)\left(x_{n}-x_{n-1}\right)=\lambda\left(x_{n+1}-x_{n}\right) .
$$

Use the Lagrange theorem, we have

$$
\left(x_{n}-x_{n-1}\right) \varphi^{\prime}\left(\bar{x}_{n}\right)+(\lambda-1)\left(x_{n}-x_{n-1}\right)=\lambda\left(x_{n+1}-x_{n}\right), \quad \bar{x}_{n} \in\left(x_{n-1}, x_{n}\right) ;
$$

or

$$
\left|x_{n+1}-x_{n}\right|=\left|\frac{\lambda-1+\varphi^{\prime}\left(\bar{x}_{n}\right)}{\lambda}\right|\left|x_{n}-x_{n-1}\right|,
$$

or, taking into account the condition (3.2),

$\left|x_{n+1}-x_{n}\right| \leq q\left|x_{n}-x_{n-1}\right|$.

From here, give the values $n=1,2, \ldots$, consistently one can obtain

$$
\begin{aligned}
& \left|x_{2}-x_{1}\right| \leq q\left|x_{1}-x_{0}\right|, \\
& \left|x_{3}-x_{2}\right| \leq q\left|x_{2}-x_{1}\right| \leq q^{2}\left|x_{1}-x_{0}\right|, \\
& \left|x_{n+1}-x_{n}\right| \leq q^{n}\left|x_{1}-x_{0}\right| .
\end{aligned}
$$

Let's consider the series

$$
x_{0}+\left(x_{1}-x_{0}\right)+\left(x_{2}-x_{1}\right)+\ldots+\left(x_{n}-x_{n-1}\right)+\ldots
$$

for which consistently approximations $x_{n}$ presented $(n+1)$ - th particular sums, i.e.

$$
x_{n}=S_{n+1} .
$$

Taking into account an inequality (3.6) the members of serious (3.7) by absolute values less with respected members of the geometrical progression by denominator $q<1$, therefore the series (3.7) converge and besides absolute. From it followed, that for continuous function $\varphi(x)$ existing the limit

$$
\lim _{n \rightarrow \infty} S_{n+1}=\lim _{n \rightarrow \infty} x_{n}=\frac{(\lambda-1) \lim _{n \rightarrow \infty} x_{n-1}+\varphi\left(\lim _{n \rightarrow \infty} x_{n}\right)}{\lambda}=\frac{(\lambda-1) \xi+\varphi(\xi)}{\lambda}=\xi,
$$

which in total is equivalent to results $\varphi(\xi)=\xi$, from which followed, that $\xi$ is the root of equation (3.5), what was require to evidence. 

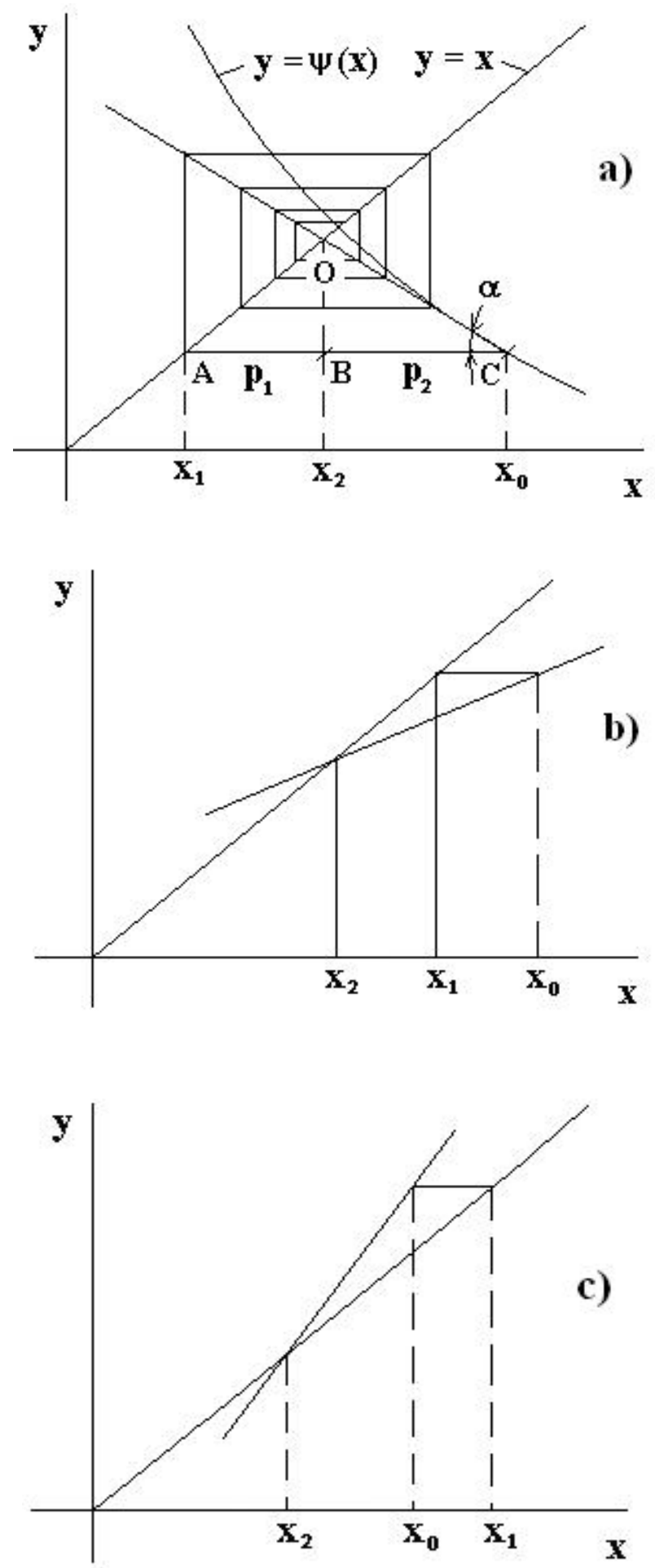

Fig.1. Geometrical interpretation of the convergence of the GMI

Let us consider the geometrical interpretation of the GMI. In a close enough to finding root $\mathrm{x}=\xi$, where allowed the assimilation of the tangent in points $\mathrm{x}_{0}, \mathrm{x}_{1}$ и $\mathrm{x}_{2}$, i.e. 
$\psi^{\prime}\left(\mathrm{x}_{0}\right)=\psi^{\prime}\left(\mathrm{x}_{1}\right)=\psi^{\prime}\left(\mathrm{x}_{2}\right)=\operatorname{tg} \alpha$, for the spiral iteration from one side carried out the fallowing equation (Fig. 1, ):

$\frac{\mathrm{BO}}{\mathrm{BC}}=\frac{\mathrm{AB}}{\mathrm{BC}}=\frac{\mathrm{p}_{1}}{\mathrm{p}_{2}}=|\operatorname{tg} \alpha|$, или $\mathrm{x}_{2}-\mathrm{x}_{1}=\left(\mathrm{x}_{0}-\mathrm{x}_{2}\right) \operatorname{tg} \alpha, \operatorname{tg} \alpha<0$.

From other side, for stage (Fig. 1, b) and hyper-stage (Fig. 1, c) iterations is just the relation:

$\mathrm{x}_{1}-\mathrm{x}_{2}=\left(\mathrm{x}_{0}-\mathrm{x}_{2}\right) \operatorname{tg} \alpha, \operatorname{tg} \alpha>0$.

Consequently, taking into account the note $\operatorname{tg} \alpha=1-\lambda$ it's justified the assumption about the existence and the singularity of a immovable point in the space $\boldsymbol{R}[4]$ and the justice of the original expression for the non linear generalization of the compression reflection (3.1), which allow to express the non linear compression operator in the space $\boldsymbol{E}$ in the finally form:

$\Phi(\mathrm{x})=\frac{\psi(\mathrm{x})-\psi^{\prime}(\mathrm{x}) \mathrm{x}}{1-\psi^{\prime}(\mathrm{x})}$.

For the cases (a) and (b) we have:

$\lambda>0 \quad\left|\frac{\lambda-1+\psi^{\prime}(x)}{\lambda}\right|<1 \Leftrightarrow\left\{\begin{array}{l}-1<\frac{\lambda-1+\psi^{\prime}(x)}{\lambda} \\ \frac{\lambda-1+\psi^{\prime}(x)}{\lambda}<1\end{array} \Rightarrow\right.$

$\Rightarrow\left\{\begin{aligned} & \psi^{\prime}(\mathrm{x})>-2|\lambda|+1 \quad\left(\text { for the spiral's iteration [ 4] at }-2|\lambda|+1<\psi^{\prime}(\mathrm{x})<0\right) \\ & \psi^{\prime}(\mathrm{x})<1 \quad\left(\text { for the step's iteration [4] at } 0<\psi^{\prime}(\mathrm{x})<1\right) .\end{aligned}\right.$

For the cases (a) and (b) we have:

$\lambda<0 \quad\left|\frac{\lambda-1+\psi^{\prime}(x)}{\lambda}\right|<1 \Leftrightarrow\left\{\begin{array}{l}-1<\frac{-\lambda-1+\psi^{\prime}(x)}{-\lambda} \\ \frac{-\lambda-1+\psi^{\prime}(x)}{-\lambda}<1\end{array} \Rightarrow\right.$

$\Rightarrow\left\{\begin{array}{cc}\psi^{\prime}(\mathrm{x})<2|\lambda|+1 ; & \text { (for a hyperstep's iteration [3] } \\ \psi^{\prime}(\mathrm{x})>1 . & \left.\text { at } 1<\psi^{\prime}(\mathrm{x})<2|\lambda|+1\right)\end{array}\right.$ 


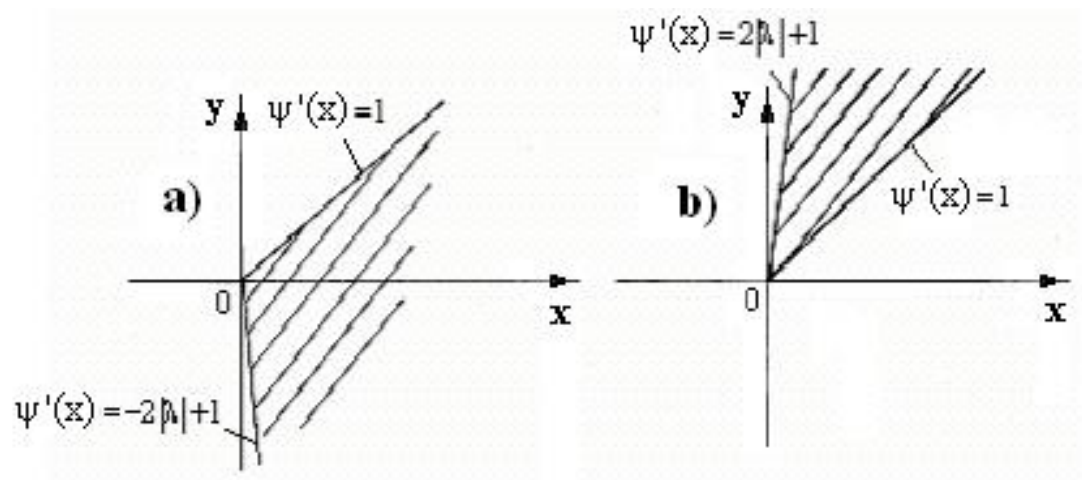

Fig.2. Representation of the sub-regions of convergence

So, from above-mentioned lay out it's obvious, that by choose of a large by absolute value number $|\lambda| \in R$, i.e. at $|\lambda| \rightarrow \infty$, all three sub-regions of convergence (Fig. 2, and b) covered practically all spectrum of a possible variation of the tangents on the plane, what required to evident. From it's followed, that the function $\Phi(\mathrm{x})$ one can to name non linear generalize compression reflection at compressing original $\psi(\mathrm{x})$ (Fig. 3).

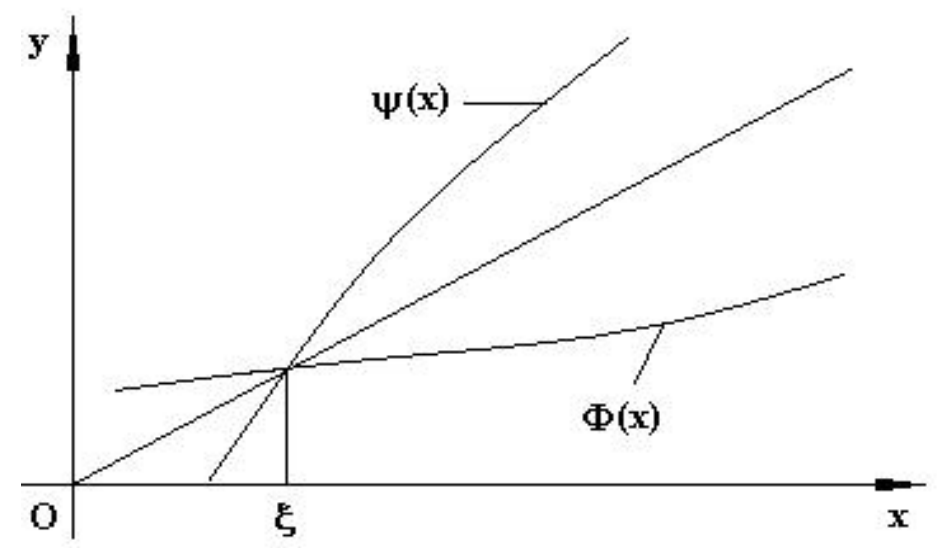

Fig.3. Non linear-generalize compression reflection $\Phi(x)$ and compressing original $\boldsymbol{\psi}(\boldsymbol{x})$

\section{NON LINEAR GENERALIZE REFLECTION AS A FUNCTION FROM SOME VARIABLES}

In the space $\boldsymbol{E}_{n}$ one introduce the canonic norm $\|\overrightarrow{\mathrm{x}}\|$, expressing one of the following norms[5]: 
$\|\overrightarrow{\mathrm{x}}\|_{\mathrm{k}}=\sqrt{\sum_{\mathrm{i}=1}^{\mathrm{n}} \mathrm{x}_{\mathrm{i}}^{2}}$, or $\|\overrightarrow{\mathrm{x}}\|_{1}=\sum_{\mathrm{i}=1}^{\mathrm{n}}\left|\mathrm{x}_{\mathrm{i}}\right|$, or $\|\overrightarrow{\mathrm{x}}\|_{\mathrm{m}}=\max _{\mathrm{i}}\left|\mathrm{x}_{\mathrm{i}}\right|$.

In this case the reflection

$\left\|\Phi\left(\mathrm{x}_{1}\right)-\Phi\left(\mathrm{x}_{2}\right)\right\| \leq \mathrm{q}\left\|\mathrm{x}_{1}-\mathrm{x}_{2}\right\| \quad$ at $\quad 0<\mathrm{q}<1$

can be qualified as a compression in the space $\boldsymbol{E}_{n}$. Consequently, for it must formally execute all known theorems [5], related to the classic compression reflection in $\boldsymbol{E}_{n}$.

On the basic of above mentioned its allowed the formalization of the compression reflection non linear operator $\vec{\Phi}$ in the space $\boldsymbol{E}_{n}$ as a operator, satisfied the compression of an arbitrary compressing original $\vec{\psi}$ to the immovable point, i.e.

$\vec{\Phi}(\vec{x})=\left[\vec{I}-\vec{\psi}^{\prime}(\vec{x})\right]^{-1}\left[\vec{\psi}(\vec{x})-\vec{\psi}^{\prime}(\vec{x}) \vec{x}\right]$

where $\vec{\psi}^{\prime}(\vec{x})$ is the Jacobean-matrix for the compressing original vector $\vec{\psi}(\vec{x})$, such as,

$$
\vec{J}(\vec{x})=\vec{\psi}^{\prime}(\vec{x})=\left[\begin{array}{cccc}
\frac{\partial \psi_{1}}{\partial \mathrm{x}_{1}} & \frac{\partial \psi_{1}}{\partial \mathrm{x}_{2}} & \cdots & \frac{\partial \psi_{1}}{\partial \mathrm{x}_{\mathrm{n}}} \\
\frac{\partial \psi_{2}}{\partial \mathrm{x}_{1}} & \frac{\partial \psi_{2}}{\partial \mathrm{x}_{2}} & \cdots & \frac{\partial \psi_{2}}{\partial \mathrm{x}_{\mathrm{n}}} \\
\dot{\frac{\partial \psi_{\mathrm{n}}}{\partial \mathrm{x}_{1}}} & \frac{\partial \psi_{\mathrm{n}}}{\partial \mathrm{x}_{2}} & \cdots & \frac{\partial \psi_{\mathrm{n}}}{\partial \mathrm{x}_{\mathrm{n}}}
\end{array}\right]
$$

\section{EXAMPLES OF THE NUMERICAL REALIZATION}

As a first example is considered the case of a numerical solution of the transcendent equation in complex roots, obtaining in [6], where the problem of stability of multilayer found was investigated: $\alpha p \operatorname{tgp}=1+i \beta p$, where $p=p_{1}+\mathrm{ip}_{2}, \quad \alpha$ and $\beta$ are parameters, receive the following values: $\alpha=0,1,10$ and $\beta=0 ; 0,1$. This equation for complex roots one can take to system of two transcendent equations for real roots: 
$\left\{\begin{array}{l}\alpha p_{1} \operatorname{tg} p_{1}-\alpha p_{2} \operatorname{thp} p_{2}=1+\beta p_{1} \operatorname{tg} p_{1} \operatorname{thp} p_{2}-\beta p_{2} \\ \alpha p_{1} \operatorname{thp} p_{2}+\alpha p_{2} \operatorname{tg} p_{1}=\beta p_{1}-\operatorname{tg} p_{1} \operatorname{th} p_{2}+\beta p_{2} \operatorname{tg} p_{1} \operatorname{th} p_{2}\end{array}\right.$

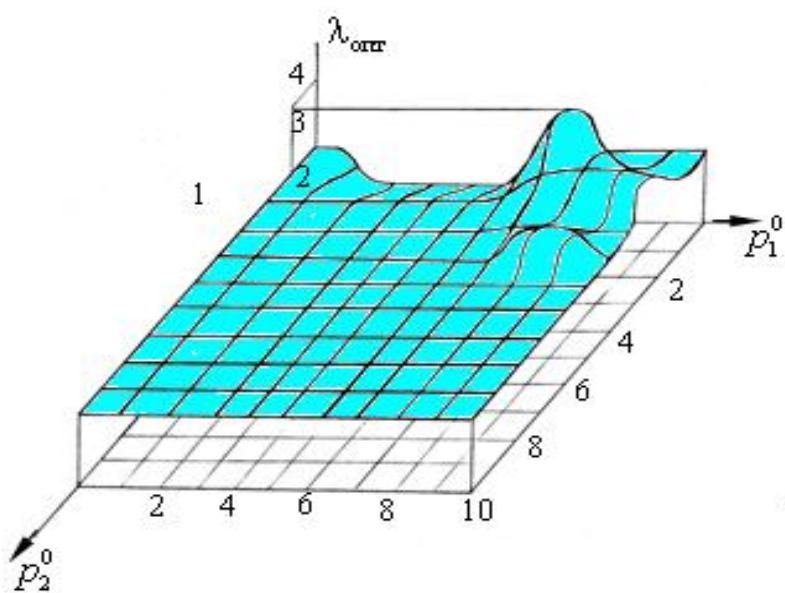

Fig.3.

Dependence of $\lambda_{\text {opt }}$ from original approximation at $\boldsymbol{\alpha}=\mathbf{0 , 1} ; \boldsymbol{\beta}=\mathbf{0}$

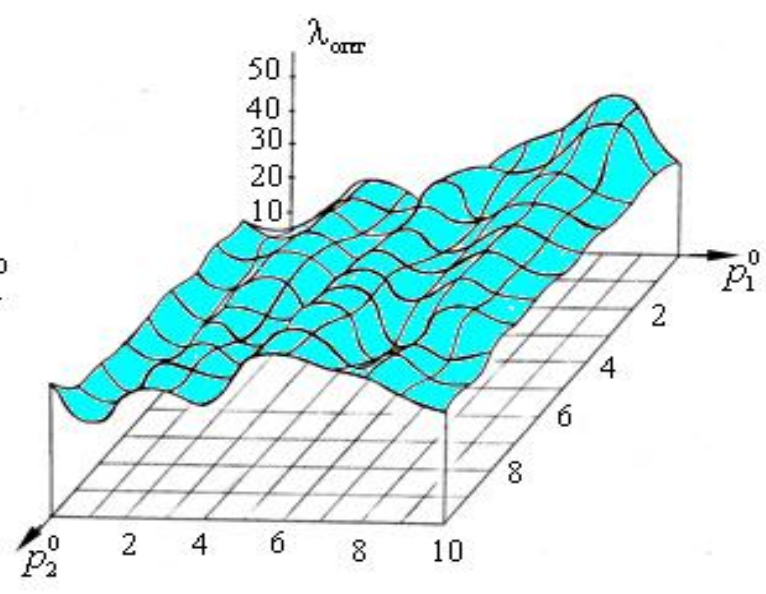

Fig.4.

Dependence of $\lambda_{\text {opt }}$ from original approximation at $\alpha=10 ; \beta=0,1$

On the Fig. 3 and Fig. 4 the optimal values of $\lambda_{\text {opt }}$ for a reach of convergence of numerical results of the mentioned example, depending from original approach $\mathrm{p}_{1}^{0}$ and $\mathrm{p}_{2}^{0}$ are brought. An exactness of the generation of numerical results: $\varepsilon=10^{-5}$. Similar tables one can obtain for arbitrary values of the pair $\alpha$ and $\beta$.

Lets take into account to the circumstance, that the attempt of a solution of the control example by Newton's and the other methods of numerical solution didn't brought to the satisfied results for roots finding.

As a second example of an algorithmic solution of the transcendent equations systems is chosen a system of quasi-transcendent equations, i.e. having an analytical solution, for some particular cases of the Collatz hypothesis [7]. This is a system of two equations, assuming at $\mathrm{k}_{0}=13, \mathrm{k}_{1}=5, \mathrm{k}_{2}=1$ a single solution in natural numbers, have the following form:

$\left\{\begin{array}{c}3 \mathrm{k}_{0}-2^{\eta_{1}} \mathrm{k}_{1}+1=0 \\ 3 \mathrm{k}_{0}+3 \mathrm{k}_{1}-2^{\eta_{1}} \mathrm{k}_{1}-2^{\eta_{2}} \mathrm{k}_{2}+2=0\end{array}\right.$ 
On the Figs. 5 and 6 a dependence of the number of iterations from original approximations of the parameters $\eta_{1}$ and $\eta_{2}$ of the above-mentioned system of equations at $\lambda=20$ and $\varepsilon=10^{-10}$ are brought.

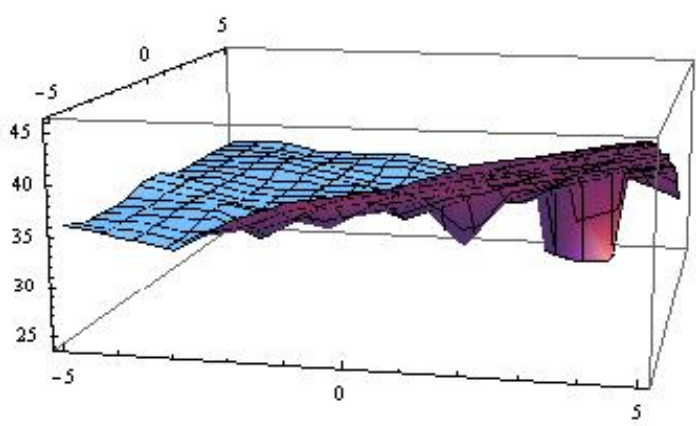

Fig.5.

Dependence of iteration numbers from original approximation by the GMI

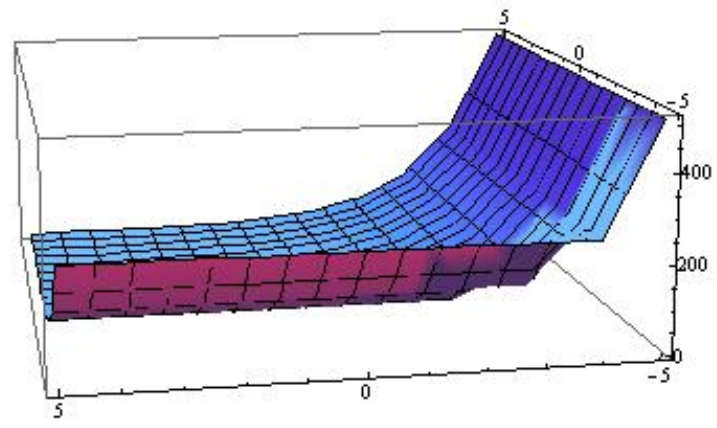

Fig.6.

Dependence of iteration numbers from original approximation by Newton method

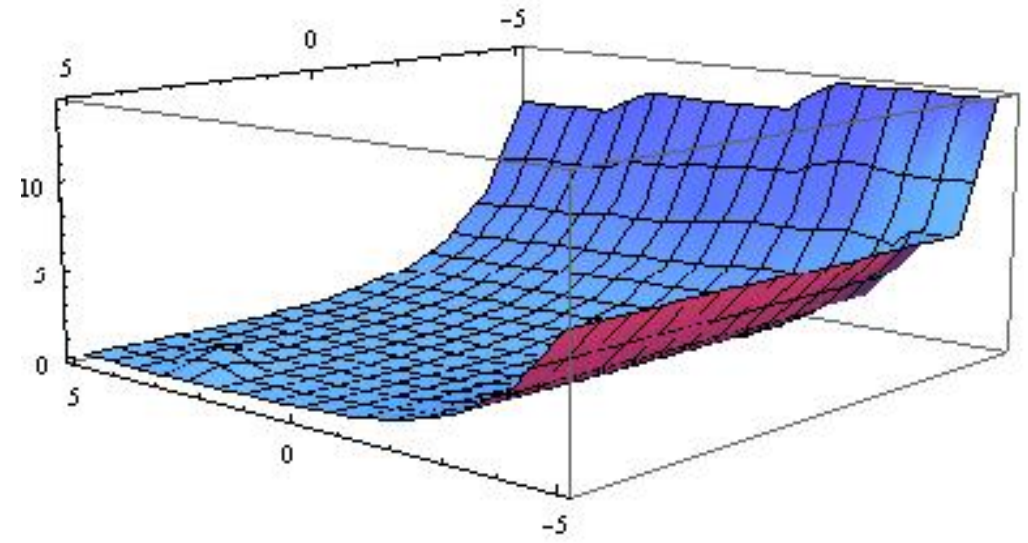

Fig.7. Relations of the iteration numbers by Newton method to the iteration numbers by the GMI

\section{CONCLUSION}

As it's shown previously theoretical foundations and above-mentioned numerical results, the GMI possess by essentially advantage with respect to the Newton and other famous calculation methods applicable to the solution of transcendent equations systems. 


\section{REFERENCES}

[1] Vantsyan . . About one method of solution of the transcendent and algebraic equations // Information Technologies and Management., 2004, 4, 44-47.

[2] Vantsyan - , Gevorgyan H.A. Modernize method of iteration // The mechanics of a composite materials and constructions, Proc. of IV - All Russian symposium, Moscow., 2012, 1, 40-44.

[3] Vantsyan . , Gevorgyan H.A. About one method solution of transcendental and algebraic equations. - Yerevan: Gitutyun., 2014, 56.

[4] Kolmogorov A.N, Fomin S.V. The elements of the theory of functions and functional analysis. - Moscow: Nauka, 1976, 542.

[5] Demidovich B.P, Maron I. . Basis of the computational mathematics, Moscow: Nauka, 1970, 664.

[6] Movsisyan L.A. About one method of the seismoprotection // Mechanics, Bulletin of the NAS RA., 2011, 111(4), 325-335.

[7] Hayes B. Table off and fall of the numbers-hailstone. - Scientific American., 1984., 3, .102-107.

\section{How to cite this article:}

Vantsyan A. A, Gevorgyan H. and Moravej M. About one non linear generalization of the compression reflection principle. J. Fundam. Appl. Sci., 2016, 8(2S), 368-379. 